Computationally Efficient Estimation of the Electric-Field Maximums for the MFT Insulation Coordination

M. Mogorovic and D. Dujic

This material is posted here with permission of the IEEE. Such permission of the IEEE does not in any way imply IEEE endorsement of any of EPFL's products or services. Internal or personal use of this material is permitted. However, permission to reprint / republish this material for advertising or promotional purposes or for creating new collective works for resale or redistribution must be obtained from the IEEE by writing to pubs-permissions@ieee. org. By choosing to view this document, you agree to all provisions of the copyright laws protecting it. 


\title{
Computationally Efficient Estimation of the Electric-Field Maximums for the MFT Insulation Coordination
}

\author{
Marko Mogorovic and Drazen Dujic \\ Power Electronics Laboratory - PEL \\ École Polytechnique Fédérale de Lausanne - EPFL \\ Station 11, CH-1015 Lausanne \\ marko.mogorovic@epfl.ch,drazen.dujic@epfl.ch
}

\begin{abstract}
This paper proposes a methodology for computationally-efficient estimation of the local E-field maximums within the transformer winding insulation material for design optimization purposes, where besides the estimation accuracy, the computational cost represents an equally important figure of merit. Except for a limited class of very simple symmetric geometries, it is not possible to analytically solve or approximate these phenomena with acceptable accuracy. While it is possible to very accurately model the electric field distribution via some computationally intensive numerical method, such as finite elements method (FEM), the execution time and numerical stability are often limiting factors when it comes to a multi-variable optimization. To that end, this paper proposes a suficiently-accurate (error $<5 \%$ referred to FEM) modeling methodology for parasitic capacitance and local E-field maximum estimation, specially designed for very fast execution - more than four orders of magnitude faster compared to FEM.
\end{abstract}

\section{NOMENCLATURE}

$C_{t 2 c} \quad$ Turn-to-core parasitic capacitance

$C_{t 2 t} \quad$ Turn-to-turn parasitic capacitance

$D_{t 2 c} \quad$ Turn-to-core distance

$D_{t 2 t} \quad$ Turn-to-turn distance

E $\quad$ Electric field

$H_{t} \quad$ Turn height

$N \quad$ Number of winding turns

$R_{t} \quad$ Turn edge radius

$V \quad$ Electric field potential

$V_{m} \quad$ Voltage pulse magnitude

$W_{t} \quad$ Turn width

$a_{i . j} \quad$ Multi-variable polynomial coefficients

$f_{i}(\mathbf{x}) \quad$ Multi-variable polynomial function

$t \quad$ Time

$\mathbf{x} \quad$ Vector of normalized variables

\section{INTRODUCTION}

Insulation coordination is an essential step in the design process of any medium (MV) or high (HV) voltage power transformer that ensures its proper and safe operation. This task is especially challenging in case of medium (MF) and high (HF) frequency transformers operating within power electronics converters - increasingly popular solid state transformer
(SST) concept [1], [2]. Whether it is a fast rising front of the basic lightning impulse (BIL) test waveform, as shown in Fig. 1a, or very fast switching of the wide band gap semiconductors, parasitic capacitances of the transformer windings will affect the voltage and electric (E)-field distribution over the winding turns during these fast transients, as displayed in Figs. $1 \mathrm{~b}$ and 2, and therefore cannot be neglected [3]-[5].

However, due to high complexity, computational cost and need for customization of FEM models, very precise models such as described in [3], [4] are not suitable for overall multi-variable design optimization, but rather for final design verification and its correction through several final iterations [6]-[9]. This paper proposes a very-fast and numericallystable estimation methodology that allows a proper inclusion of the insulation coordination considerations within the design optimization process.

In this work, for the purpose of design optimization, the BIL is approximated with a Heaviside step function as a worst case scenario, as displayed in Fig. 1a. This approximation allows for the reduction of the complex $\mathrm{HF}$ transformer winding model to a network of parasitic capacitances, as shown in Fig. 3a, which can be analytically solved in closed form, as given in

$$
V(i)=V_{m} \frac{\sinh (\alpha i)}{\sinh (\alpha N)} \quad \text { where } \quad \alpha=\sqrt{\frac{C_{t 2 c}}{C_{t 2 t}}}
$$

where $i$ is the winding turn index, $N$ is the total number of turns and $C_{t 2 c}$ and $C_{t 2 t}$ are the turn-to-core and turn-to-turn parasitic capacitances, respectivelly.

Voltage and E-field distribution over the transformer winding excited with Heaviside step function, at the moment of the pulse incidence $(t=0)$, are shown in Figs. $2 \mathrm{a}$ and $2 \mathrm{~b}$, respectively. It can be seen that the initial voltage distribution over the winding turns $\left(V_{0}\right)$ is uneven - first turns are experiencing turn-to-turn voltages drastically above the nominal value. Moreover, some turns experience high, above-nominal, absolute voltage levels during the fast transient on natural frequency of the winding $\left(V_{t . \max }\right)$. This causes high local Efield magnitude-peaks that may lead to gradual (or in extreme 


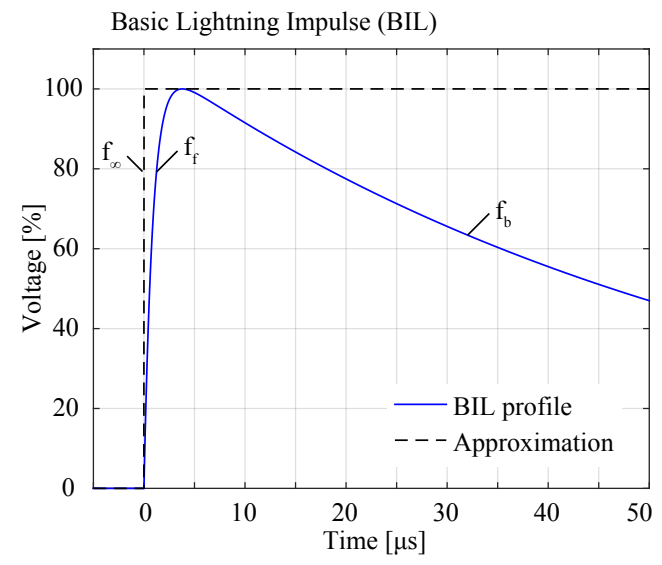

(a)

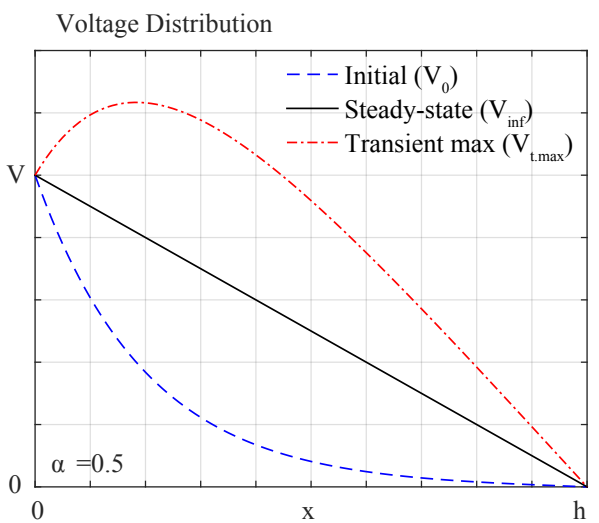

(b)

Fig. 1. (a) Standard $1.2 \times 50 \mu$ s full-wave lightning impulse profile, according to IEEE Std 4-1995 and IEEE Std C57.98-1993 (equivalent frequencies of the fast rising front and slow descending back are $f_{f}=200 \mathrm{kHz}$ and $f_{b}=5 \mathrm{kHz}$, respectively). (b) Example of the theoretical envelope of the voltage distribution over the transformer winding turns during fast "ringing" transients where: $V_{0}$ is the initial voltage distribution at $t=0$; $V_{t m}$ is the maximum theoretical turn voltages during subsequent "ringing" transient; $V_{i n f}$ is the voltage distribution after the fast transient response has transpired;

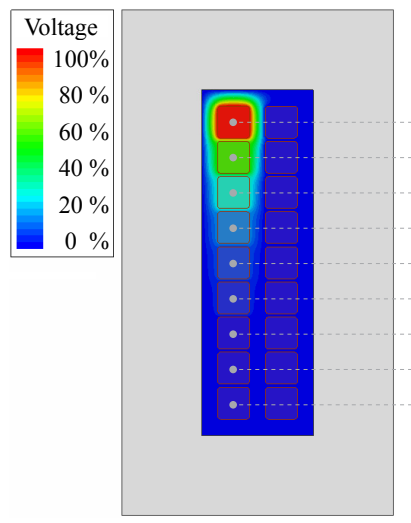

(a)

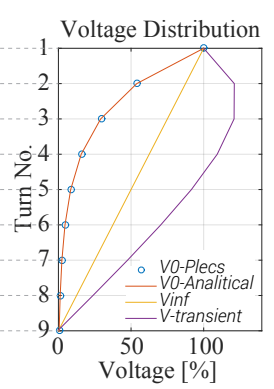

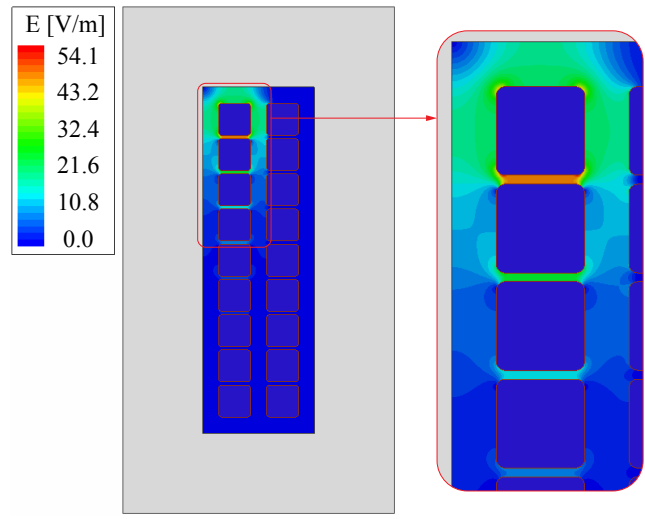

(b)

Fig. 2. 2D electrostatic FEM simulation of a representative 2-winding MFT cross-section example, excited with a theoretical BIL approximation (Heaviside step function) at the moment of incidence $(t=0)$ : (a) voltage distribution and (b) E-field distribution. For the sake of simplicity, a single layer winding structure is considered.

cases instant) annihilation of the insulation material if not properly accounted for. These effects are well known from the traditional line frequency transformer and electric machine design. Good understanding of main parameters governing this phenomena is necessary for the proper insulation coordination. From a design point of view, reliable modeling is paramount in order to avoid massive and costly over-sizing.

\section{Proposed Modeling}

A detailed electrostatic finite elements method (FEM) analysis is performed to identify the critical regions, where the insulation material is experiencing the highest dielectric stress. As can be seen in Fig. 2b, the maximum local E-field magnitude peaks are occurring somewhere along the conductor edge. These local E-field maximums are a function of the geometry and voltage distribution. On the other hand, assuming the Heaviside step excitation, the voltage distribution is purely a function of turn-to-core and turn-to-turn parasitic capacitances, which again depend only on the geometry. Therefore, in order to predict the maximum local E field peaks, it is necessary to:

(i) Model the parasitic capacitances, based on the known design geometry

(ii) Solve the parasitic capacitance network to obtain the voltage distribution

(iii) Model the local E-field maximum, based on the known geometry and voltage distribution

While (ii) boils down to a simple evaluation of (1) in case of Heaviside step-function excitation, (i) and (iii) remain numerically challenging. The remainder of this paper, describes in detail the proposed methodology for a very fast estimation of these values - the statistical data-driven models (SDDMs) derived based on extensive FEM simulation.

A full parasitic capacitance network can be seen in Fig. 3a. Taking into account that both the core and secondary winding are at the ground potential, the secondary winding can also 


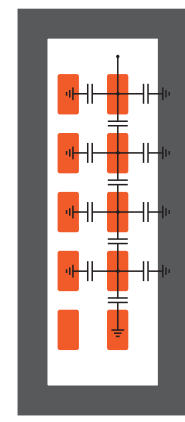

(a)

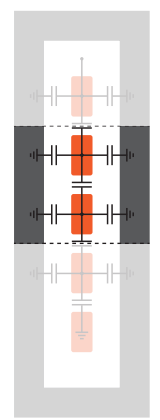

(b)

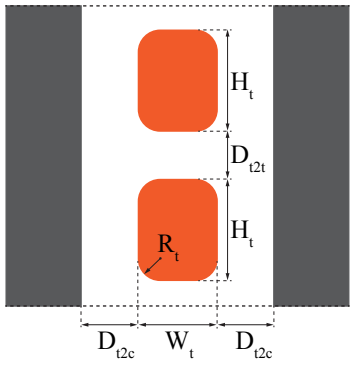

(c)

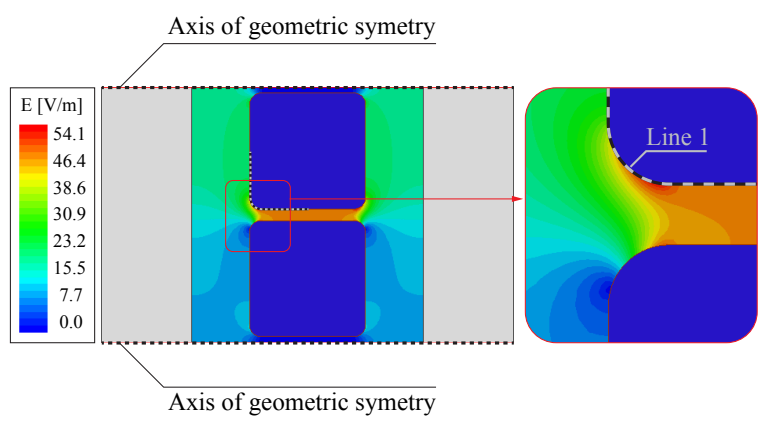

(d)

Fig. 3. (a) Full parasitic capacitance model of a generalized MFT geometry cross-section. (b) Simplified geometry equivalence. (c) Minimal generalized geometry detail. (d) 2D electrostatic FEM simulation of the minimal generalized geometry detail.

TABLE I

Definition ANd RANGe of Normalized PARAmeters ACCORDing To Fig. 3C

\begin{tabular}{lccccc}
\hline Norm. Vars. & $x_{1}$ & $x_{2}$ & $x_{3}$ & $x_{4}$ & $x_{5}$ \\
Definition & $\frac{H_{t}}{W_{t}}$ & $\frac{D_{t 2 t}}{W_{t}}$ & $\frac{D_{t 2 c}}{H_{t}}$ & $\frac{R_{t}}{\min \left(H_{t}, W_{t}\right)}$ & $\frac{U_{t 2 t}}{U_{t 2 c}}$ \\
Range [p.u.] & $0.25-4$ & $0.01-4$ & $0.01-4$ & $0.02-0.5$ & $0-1$ \\
\hline
\end{tabular}

Note that these are very extensive ranges, covering most of designs

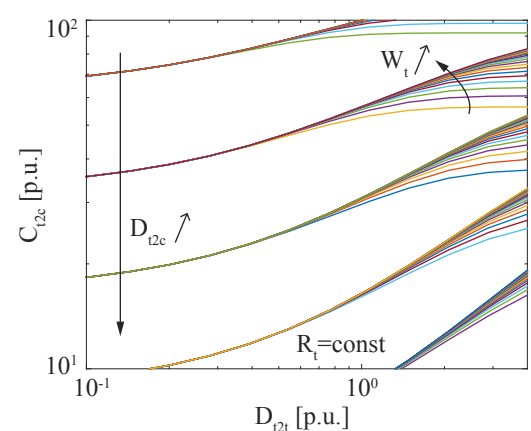

(a)

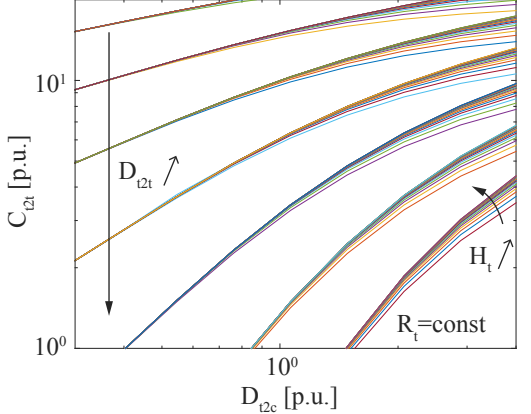

(b)

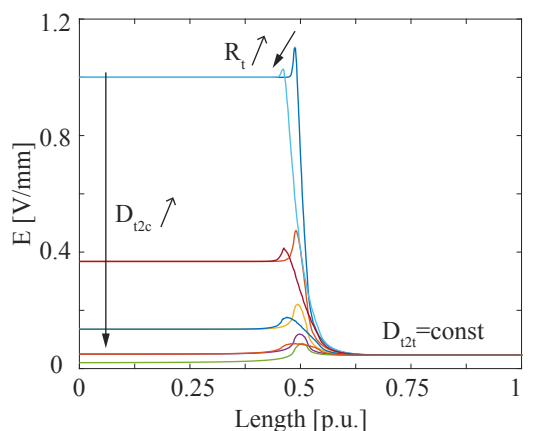

(c)

Fig. 4. Plots of families of curves, exposing the effects of various parameters, as given in Table I, on: (a) turn-to-core $C_{t 2 c}=f_{1}\left(x_{1}, x_{2}, x_{3}, x_{4}\right)$, (b) turn-to-turn $C_{t 2 t}=f_{2}\left(x_{1}, x_{2}, x_{3}, x_{4}\right)$ parasitic capacitances and (c) the local E-field magnitude peak along the edge of the turn conductor $E_{m a x}=f_{3}\left(x_{2}, x_{3}, x_{4}, x_{5}\right)$, as highlighted with Line 1 in Fig. $3 \mathrm{~d}$.

be seen as a straight wall boundary at the ground potential, as shown in Fig. 3b. Note that, all conductive parts of the transformer, including any other winding except the one being excited by the test pulse, are grounded at all terminals during the BIL test, as recommended by IEEE Std 4-1995 and IEEE Std C57.98-1993. Finally, based on the geometric symmetry and periodic structure of the winding, a minimal generalized geometry detail, capable of capturing all of the phenomena of interest, is identified and parametrised, as given in Fig. $3 \mathrm{c}$ and Table I.

A 2D FEM parametric sweep is performed on the generalized geometry detail (Fig. 3d), as defined in Table I, extracting the turn-to-core and turn-to-turn parasitic capacitances and maximum local E-field peaks within the parameter ranges of interest. Sample plots of this data, illustrating the effects of various parameters on the modeled values are shown in Fig. 4.
A multi-variable polynomial fitting is performed on these data sets, thus generating the following SDDM models

$$
\begin{gathered}
C_{t 2 c}=f_{1}\left(x_{1}, x_{2}, x_{3}, x_{4}\right) \\
C_{t 2 t}=f_{2}\left(x_{1}, x_{2}, x_{3}, x_{4}\right) \\
E_{\text {max }}=f_{3}\left(x_{2}, x_{3}, x_{4}, x_{5}\right)
\end{gathered}
$$

with inherent high accuracy of FEM simulations and very low computational cost, characteristic for simple arithmetic operations (matrix multiplications) needed for evaluation of polynomials.

Taking into account the minimum vector of influential variables $(\mathbf{x})$, as summarized in Table $\mathrm{I}$, a multi-variable polynomial fitting is done, variable by variable, as displayed in Fig. 5, allowing to always choose the optimal order of polynomial, while inherently ensuring convergence [10]. The 


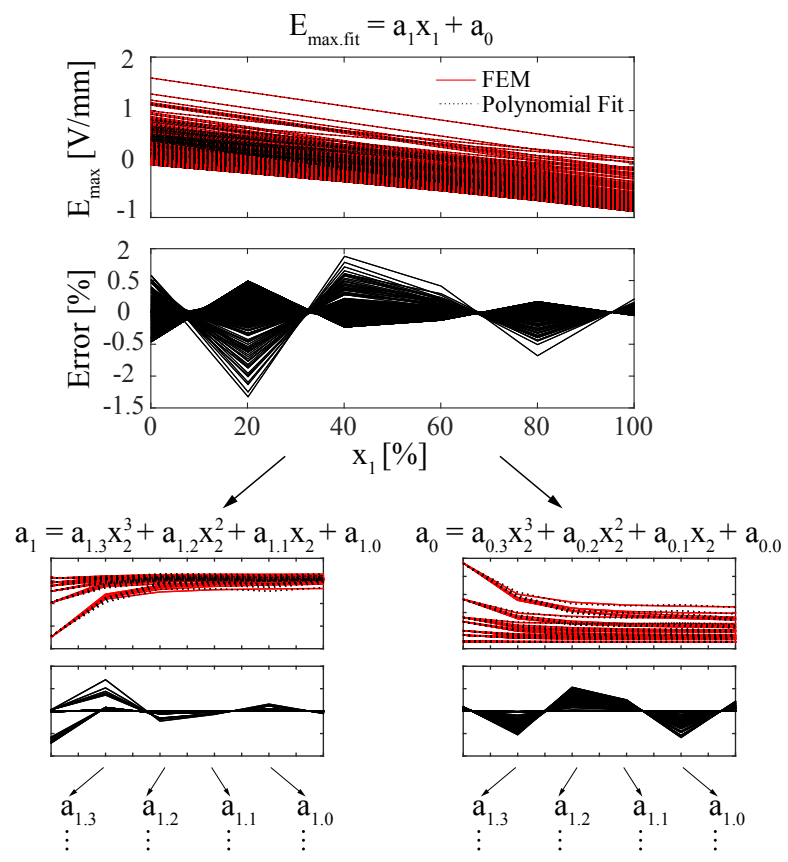

Fig. 5. An example of the employed multi-variable polynomial fitting methodology; fitting of the local E-field maximum $\left(E_{\max }\right)$ to the results of the 2D FEM sweep (50'000 simulations), according to Fig. 3d

final result of this fitting is a multi-dimensional array of polynomial parameters. For the sake of illustration, an example of the described model, for a two variable case is shown in (5).

$$
E_{\text {max.fit }}=\left[\begin{array}{c}
x_{1} \\
1
\end{array}\right]^{T}\left[\begin{array}{llll}
a_{1.3} & a_{1.2} & a_{1.1} & a_{1.0} \\
a_{0.3} & a_{0.2} & a_{0.1} & a_{0.0}
\end{array}\right]\left[\begin{array}{c}
x_{2}^{3} \\
x_{2}^{2} \\
x_{2} \\
1
\end{array}\right]
$$

As can be seen, the evaluation of the model boils down to simple low order matrix multiplications which execute very fast on the processor. Depending on how many variables $(N)$ are involved, and what are the orders of the polynomial fittings $\left(n_{i}\right)$, there is $\prod_{i=1}^{N} n_{i}$ polynomials to be solved. In case of 4variables, as described by Fig. 3c, Table I, and Fig. 4, these models execute more than four orders of magnitude faster than FEM. For the sake of illustration, $120^{\prime} 000$ 2D FEM simulations of the local $\mathrm{E}$ field maximum, requiring a fine mesh in the conductor edge regions, take almost full nine days to execute, while the same $120^{\prime} 000$ estimations with SDDM execute in 73 seconds on a same computer.

All three models ((2), (3) and (4)) are derived under assumption of surrounding vacuum. A different insulation material can easily be taken into account with a simple multiplication/division with its relative permittivity $\varepsilon_{r}$. Moreover, note that the formula for local E-field maximum is a function of the voltage distribution and therefore covers all relevant voltage distributions that may be encountered during normal $\left(V_{i n f}\right)$ or transient operation $\left(V_{0}\right.$ and $\left.V_{t . \max }\right)$.

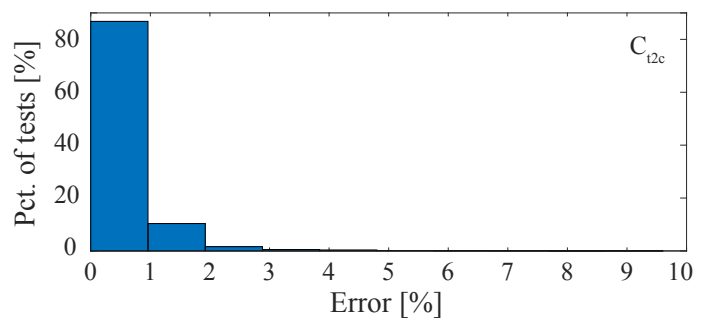

(a)

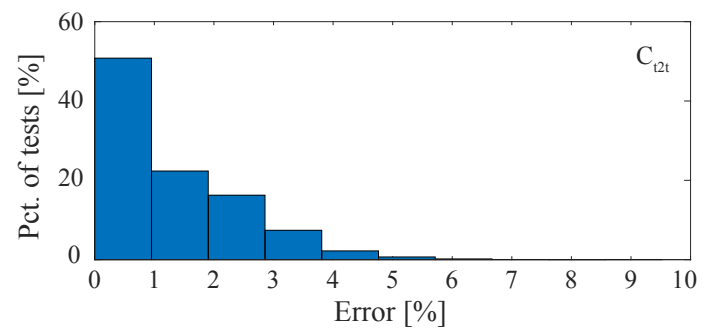

(b)

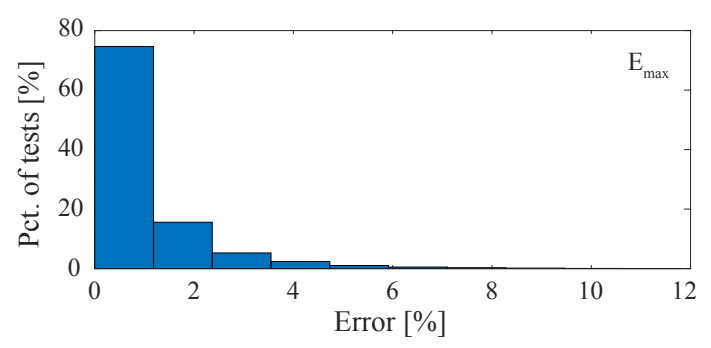

(c)

Fig. 6. Relative estimation error histograms of the proposed data-driven statistical models for computation of: (a) turn-to-core parasitic capacitance, (b) turn-to-turn parasitic capacitance and (c) maximum local E-field magnitude peak.

Relative estimation errors of the three aforementioned multivariable polynomial models referred to the FEM simulation results are given in Fig. 6. It can be seen that a very good accuracy can be achieved, with errors practically below $5 \%$, while decreasing the execution time four orders of magnitude compared to FEM. (1)-(4) together formulate a framework for a computationally efficient estimation of the local Efield maximums allowing the study of separate influences on insulation coordination and overall design optimization.

While these formulas fully cover the simplest case of single layer windings, discussed in this wok, additional formulas would have to be derived to take into account more complex cross-layer turn-to-turn parasitic couplings of the multi-layer winding structures. Finally, while these models facilitate the inclusion of many insulation coordination considerations into design optimization, they are still a simplified representation of the transformer and a detailed 3D FEM analysis is still advised as a final verification prior to prototyping.

\section{EXPERIMENTAL VERIFICATION}

For the purposes of experimental verification, a multiwinding transformer prototype has been realized, with the 


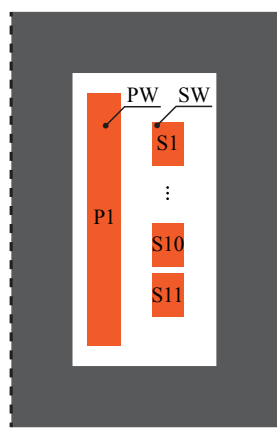

(a)

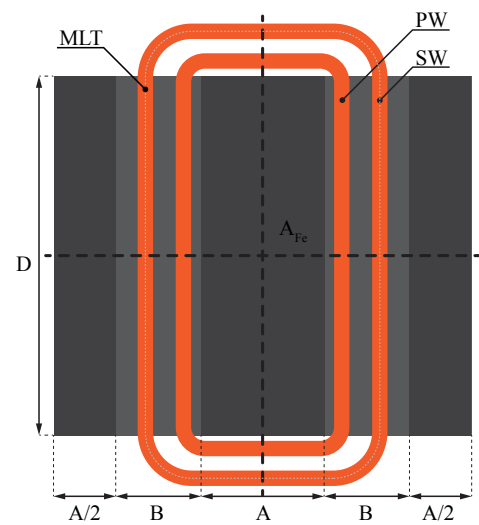

(b)
Fig. 7. Illustration of the winding arrangement of the multi-winding transformer prototype for the experimental verification, as shown in Fig. 8: (a) Vertical cross-section and (b) Horizontal cross-section

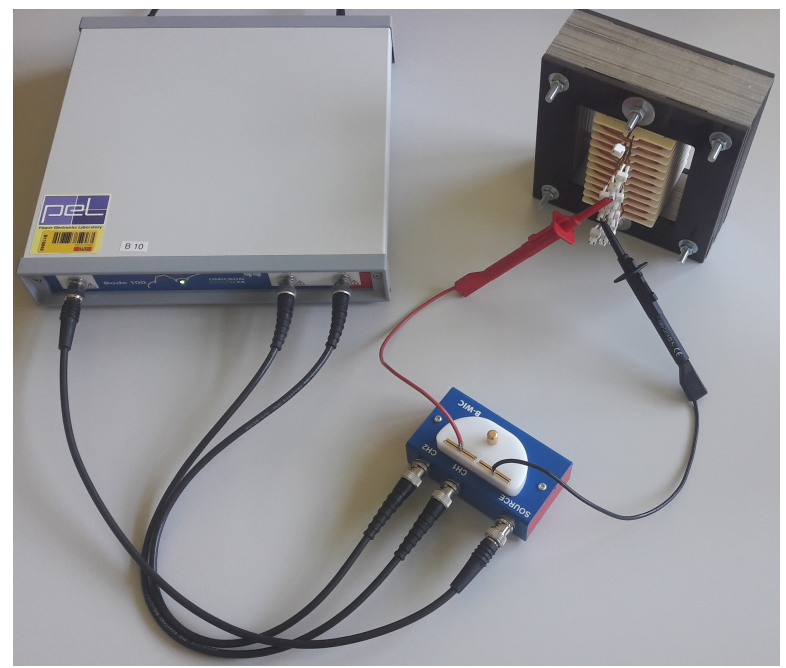

Fig. 8. Multi-winding transformer test setup for experimental verification

winding arrangement, as displayed in Fig. 7 - a single primary and the secondary winding split into 11 partitions. While it is not possible to obtain the measurements of the local electric field peaks, the parasitic capacitance models can be verified on such a prototype.

Unfortunately, the separate parasitic couplings, as described by (2) and (3) cannot be directly measured. Only the total capacitance including all of the couplings can be experimentally obtained between any two elements. Bode 100 vector network analyzer is used to measure the total parasitic capacitance between each secondary and the primary winding, as well as between each secondary and the middle (6th) secondary winding of the multi-winding transformer prototype, as given in Fig. 8.

The estimated value of these total coupling parasitic capacitances can be obtained from the total winding parasitic capacitance network, as shown in Fig. 3a, using the Krone
Secondary-to-Ground Parasitic Capacitance [pF]

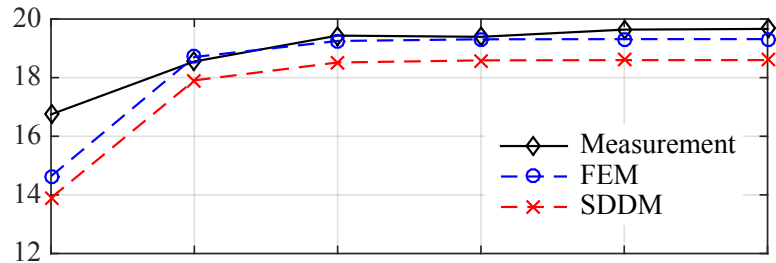

Estimation Error [\%]

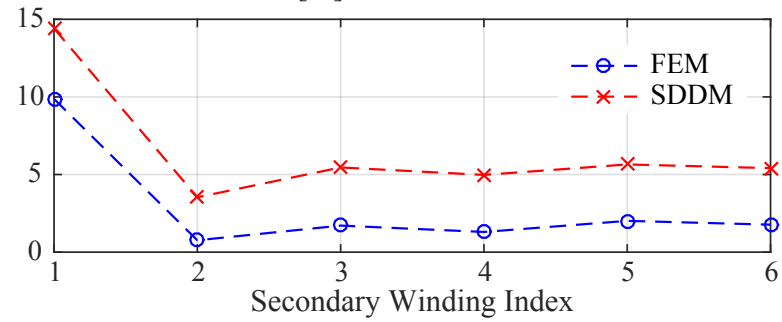

(a)
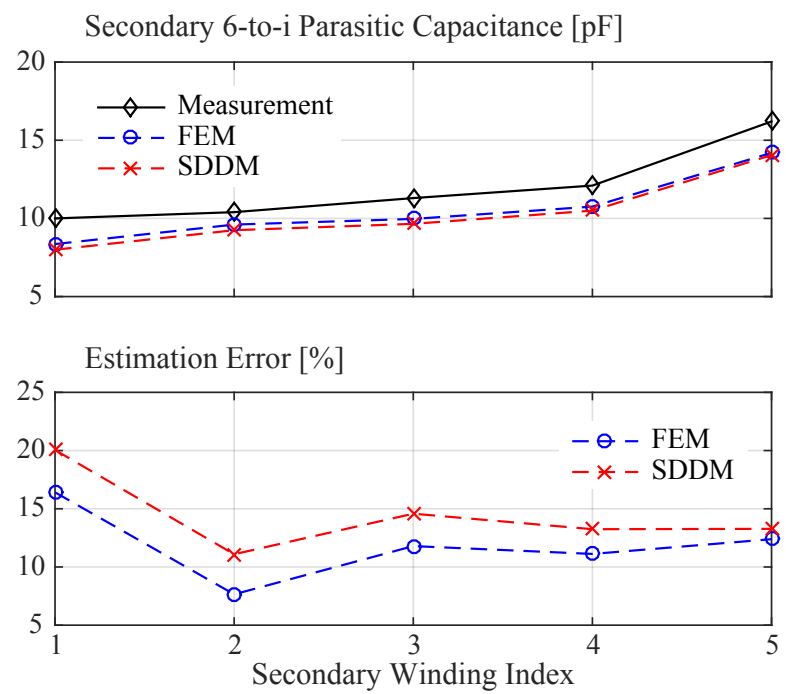

(b)

Fig. 9. Relative estimation error histograms of the proposed data-driven statistical models for computation of: (a) turn-to-core parasitic capacitance, (b) turn-to-turn parasitic capacitance and (c) maximum local E-field magnitude peak.

reduction on its admittance matrix. With this operation the total capacitance matrix can be reduced to one equivalent capacitance between any two given nodes. These total parasitic couplings are a function of the separate parasitic couplings described by (2) (turn-to-core and turn-to-primary winding) and (3) (turn-to-turn) and therefore only as accurate as the associated SDDM models.

The plots of the aforementioned total secondary-to-primary and secondary-to-secondary parasitic capacitance measurements and their estimation are shown in Fig. 9. It can be seen that, as expected, the SDDM models are within the $5 \%$ error range compared to FEM results. Moreover, the relative error referred to the measurement results is below $20 \%$ which is a 
good result considering the very low value and sensitivity of these parameters.

\section{CONCLUSION}

Proper insulation coordination of the transformer is paramount for its proper and safe operation. On the other hand, the associated modeling of the dielectric stress due to the local electric field peaks is a challenging task. Even more so when it comes to design optimization where the execution speed of the model is an important figure of merit. This paper proposes a computationally efficient, yet sufficiently accurate, statistical data-driven model of the local electric field maximums within the insulation of the transformer windings, based on FEM simulation of simple geometry details and mutivariable polinomial fitting.

The proposed SDDM framework has been described in detail. It was shown how it is possible to transform, generalize and normalize a numerically difficult problem up to the point where it can be very efficiently captured via a standardized multi-variable polynomial model. The obtained three SDDM models execute more than four orders of magnitude faster, while achieving the the accuracy within $5 \%$ error range compared to FEM. Moreover, given the very low value and sensitivity of the given parameters, a relatively good estimation accuracy of the total parasitic capacitance coupling is achieved, with errors less than $5 \%$ relative to FEM and less than $20 \%$ relative to the measurement.

Even beyond this specific study, this new class of models, optimized for the best trade-off between accuracy and execution speed will enable further inclusion of very sophisticated simulations, with otherwise impractically high computational cost, into design optimization process, resulting in cutting edge performance.

\section{REFERENCES}

[1] J. E. Huber and J. W. Kolar, "Solid-State Transformers: On the Origins and Evolution of Key Concepts," IEEE Ind. Electron. Mag., vol. 10, no. 3, pp. 19-28, Sep. 2016.

[2] M. Claessens, D. Dujic, F. Canales, J. K. Steinke, P. Stefanutti, and C. Vetterli, "Traction Transformation: A Power-Electronic Traction Transformer (PETT)," ABB Review, No: 1/12, pp. 11-17, 2012.

[3] J. Smajic, T. Steinmetz, M. Rüegg, Z. Tanasic, R. Obrist, J. Tepper, B. Weber, and M. Carlen, "Simulation and measurement of lightningimpulse voltage distributions over transformer windings," IEEE Transactions on Magnetics, vol. 50, no. 2, pp. 553-556, Feb. 2014.

[4] T. Župan, B. Trkulja, R. Obrist, T. Franz, B. Cranganu-Cretu, and J. Smajic, "Transformer windings' $r l c$ parameters calculation and lightning impulse voltage distribution simulation," IEEE Transactions on Magnetics, vol. 52, no. 3, pp. 1-4, Mar. 2016.

[5] T. Appel and D. Benner, "Fundamental Investigation of very fast Transients in Power-Electronic Winding for Solid-State Transformers," in 19th European Conference on Power Electronics and Applications (EPE'17 ECCE Europe), Warsaw, Poland, 2017., pp. 1-10.

[6] T. Gradinger, U. Drofenik, and S. Alvarez, "Novel Insulation Concept for an MV Dry-Cast Medium-Frequency Transformer," in 19th European Conference on Power Electronics and Applications (EPE'17 ECCE Europe), Warsaw, Poland, 2017., pp. 1-10.

[7] G. Ortiz, "High-Power DC-DC Converter Technologies for Smart Grid and Traction Applications," PhD thesis, ETH Zurich, Switzerland, 2014.

[8] M. Mogorovic and D. Dujic, "100 kW, $10 \mathrm{kHz}$ Medium-Frequency Transformer Design Optimization and Experimental Verification," IEEE Transactions on Power Electronics, vol. 34, no. 2, pp. 16961708, Feb. 2019.

[9] _ - "Sensitivity analysis of medium-frequency transformer designs for solid-state transformers," IEEE Transactions on Power Electronics, vol. 34, no. 9, pp. 8356-8367, Sep. 2019.

[10] _ _Computationally Efficient Leakage Inductance Estimation of Multi-Winding Medium Frequency Transformers," in PCIM Europe 2019; International Exhibition and Conference for Power Electronics, Intelligent Motion, Renewable Energy and Energy Management, May 2019, pp. 1-6. 